\title{
Least Coupling Paths Model for Non-contact EMI base on Lump Element Approach in Switch Mode Converter
}

\author{
N.K. Poon C.P. Liu M.H. Pong \\ Power Electronics Lab., EEE Dept., The University of Hong Kong, Hong Kong. \\ http://www.eee.hku.hk/power electronics lab/ \\ nkpoon@eee.hku.hk, cpliu@eee.hku.hk, mhp@eee.hku.hk
}

\begin{abstract}
A simplified physical model concludes a minimum of six equivalent physical coupling paths, Least Coupling Paths ( $L C P$ ), using lump elements approach through non-contact EMI coupling mechanism to the terminals of LISN. Simulation shows both inductive and capacitive coupling through each path may produce substantial EMI measured by a LISN. This physical model is good for understanding, diagnosis and emission estimation.
\end{abstract}

\section{INTRODUCTION}

The real EMI behavior of a converter is very complicated. It is not practical to input 3-D physical construction of each component, PCB layout, and operating condition into a computer to simulate EMI performance by solving the Maxwell Equation numerically. It is more comprehensible to use lump element approach to analyze the EMI performance as it can be derived from the existing circuit. It is more convenient to analyze and the result can be produced by common circuit simulation program.

Modeling EMI behavior of a switching converter by lump element approach is not new and was presented by a lot of engineers [1-9]. Engineer usually select one significant path according to their understanding and simplify the work. However different people use different simplified model to predict or explain the result. Simplifying circuit is an usual engineering practice but there needs work to show whether a simplified circuit is valid or not.

A systematic approach is proposed. We find that there exist a minimum of six coupling paths, which form a physical Least Coupling Paths $L C P$ model which models the interface between the converter and the terminals of the LISN. These paths contribute to conducted noise produced through non-contact paths where non-contact paths are defined as the paths which cannot be found on the converter circuit diagram. Experiment and simulation show that each path may contribute substantial noise at the LISN. This $L C P$ model is comprehensible and easy implement. It can be easily obtained by modifying a converter circuit consisting of dominated noise source.

\section{LUMP ELEMENTS MODEL}

\section{II.I Least Coupling Paths}

The merit of lump element approach for EMI modeling is that this approach combines lump elements for EMI with the converter circuit to facilitate circuit analysis. The assumptions made are a) only lump circuit elements are used to build the circuit model, b) the circuit consists of a number of voltage nodes and current loops.

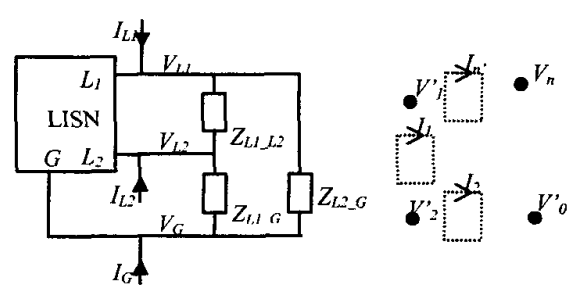

(a) $m=n$

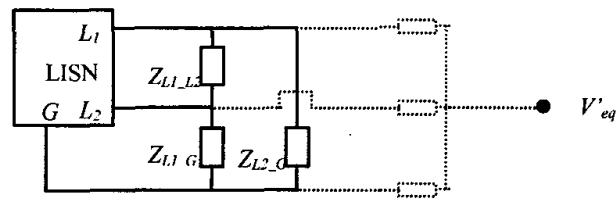

(b) $\mathrm{m}=0$

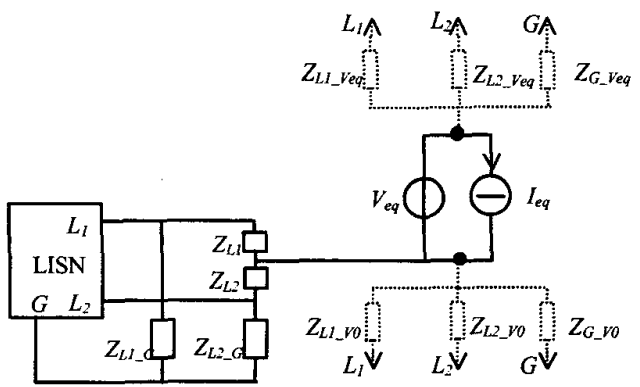

(c) $\mathrm{m}=1$

Fig. 1 Simplified $L C P$ models of a converter. a) $m=n$, general model for non-contacted noise network b) $m=0$, one equivalent voltage node only c) $m=1$, physical model for choosing two arbitrary voltage node $V_{e q}^{\prime}$ and $V_{o}$.

Fig. 1a shows a general view of a converter network with non contact coupling paths linking an LISN. Zero potential lies at infinity and within $V_{i} . n+l$ voltage nodes lie inside the converter which induce non-contact current flow to the three terminals of LISN. A number of current loops $I_{i}$ are also present in the converter. The voltages at the LISN terminals $L_{1}, L_{2}$ and $G$ are defined as $V_{L 1}, V_{L 2}$ and $V_{G}$ respectively. The equivalent input impedance of the converter between $L_{1}-L_{2}, L_{I}-G$ and $L_{2}-G$ are defined as, $Z_{L I_{-} L 2}, Z_{L I_{-} G}$ and $Z_{L L_{-} G}$ respectively. 
We define the coupled currents flowing to $L_{1}, L_{2}$ and $L_{G}$ to be $I_{L 1}, I_{L 2}$, and $I_{G}$ respectively. The coupled currents are the summation of current generated from each node.

In order to simply the circuit shown in Fig. 1a, a lesser number of fictitious nodes are created to replace the actual $n+1$ nodes. Here $m+l$ fictitious nodes are created such that

$$
\begin{aligned}
& I_{L 1}=\sum_{i=0}^{n} \frac{V_{i}-V_{L 1}}{Z_{L 1_{-} i}}=\sum_{j=0}^{m} \frac{V_{j}-V_{L 1}}{Z_{L 1_{-} V j}} \\
& I_{L 2}=\sum_{i=0}^{n} \frac{V_{i}-V_{L 2}}{Z_{L 2_{-} i}}=\sum_{j=0}^{m} \frac{V_{j}-V_{L 2}}{Z_{L 2_{-} V j}} \\
& I_{G}=\sum_{i=0}^{n} \frac{V_{i}-V_{G}}{Z_{G_{-} i}}=\sum_{j=0}^{m} \frac{V_{j}-V_{G}}{Z_{G_{-} V j}}
\end{aligned}
$$

Usually $m \leq n$ and starting from $m=0$

$$
\begin{array}{ll}
m=0 & \begin{array}{l}
\text { rejected, as no return path is formed as } \\
\text { shown in Fig. } 1 \mathrm{~b}
\end{array} \\
m=1 & \begin{array}{l}
\text { accept, as complete path is formed to } \\
\text { generate } I_{L 1}, I_{L 2} \text { and } I_{G} .
\end{array}
\end{array}
$$

Starting from one node with $m=0$. Here although a circuit can be drawn as shown in Fig. 1b, the simplified circuit shows no return path for the one single voltage node. The only solution is $I_{L 1}=I_{L 2}=I_{G}=0$, or $V_{e q}^{\prime}=V_{L 1}=V_{L 2}=$ $V_{G}$. Hence one single node with impedance connected to input lines is insufficient to form a model.

A complete circuit can be formed when $m=1$, or two nodes having impedances connected to the input lines. The following shows non-homogenous equations describing the model,

$$
\begin{aligned}
& I_{L 1}=\frac{V_{e q}^{\prime}-V_{L 1}}{Z_{L 1_{\_} V e q}}+\frac{V_{0}-V_{L 1}}{Z_{L 1_{-} V 0}}, \\
& I_{L 2}=\frac{V_{e q}^{\prime}-V_{L 2}}{Z_{L 2_{-} V e q}}+\frac{V_{0}-V_{L 2}}{Z_{L 2_{-} V 0}}, \\
& I_{G}=\frac{V_{e q}^{\prime}-V_{G}}{Z_{G_{-} V e q}}+\frac{V_{0}-V_{G}}{Z_{G_{-} V 0}},
\end{aligned}
$$

As shown from (4),(5),(6), $V_{e q}, V_{0}, Z_{L L_{-} V e q}, Z_{L 2_{-} V e q}$, $Z_{G_{-} V e q}, Z_{L_{-} V 0}, Z_{L_{-} V 0}$ and $Z_{G_{-} V 0}$ are variable and only any three of them can be solved, and other becomes arbitrary selected.

Now we choose voltage node $V_{0}$ to be a " $0 \mathrm{~V}$ " of the original circuit in order to form a closed circuit diagram. This provides the potential difference between elements to eliminate the need to consider the absolute zero voltage at infinity.
Fig. 1c now gives a more "friendly" look model with one "dominated" noise source $V_{e q}=V_{e q}^{\prime}-V_{0}$ connected to the " $0 \mathrm{~V}$ " of the circuit. Two equivalent series impedances $Z_{L I}$ and $Z_{L 2}$ forms the general $\mathrm{AC}$ input impedances between input lines and " $0 \mathrm{~V}$ " of the circuit. $Z_{L I}$ and $Z_{L 2}$ are not necessarily equal, in fact it is not equal in most converter or operating condition which cause unbalanced current as reported by [4].

The EMI model with only one dominated noise source is widely used by engineer to eliminate the complexity of circuit analysis. However, Fig. 1c shows there are at least six impedance, $Z_{L_{-} \text {Veq }}, Z_{L 2_{-} \text {Veq }}, Z_{G_{-} V e q}, Z_{L I_{-} V 0}, Z_{L 2_{-} V 0}$ and $Z_{G_{-} V 0}$. linked between voltage source $V_{e q}$ and all three terminals of the LISN. Those six impedance forms a minimum of six $L C P$ path which should be considered from an engineering point of view.

There are two reasons for choosing $m=1$, or two fictitious nodes, to form the $L C P$ model, although different number of $m$ can also be used to generate other models. First, when $m=l$, it forms the equivalent circuit with minimum noise source caused by all voltage nodes. Secondly, only one noise source is good for understanding of a switching converter, which really has one dominant noise source, as observed through the original circuit.

One can also draw some notes when the $L C P$ model is used. As a complete circuit is made by $L C P$ modelling, there is no need to distinguish between the common terms of "differential mode current" and "common mode current." All non-contact noise voltage across the terminals of LISN are caused by the noise current flowing from the six paths in all dimensions as mentioned. In fact, it is quite wrong to define which current is differential mode or common mode, as all noise current flowing into LISN are affected by the whole network and noise voltage.

Although the analysis of the EMI noise picked up by the LISN becomes a straight forward circuit analysis issue if all the variables were solved, this model do not provide an advantage to find out the exact value of all variables easily. It still needs to know all the parasitic capacitance and its corresponding voltage to solve the system equation. Nevertheless, the merit of this model is very good for approximation, especially in switching converter with one or two dominant noise voltage nodes.

Also, it is believed that this model can help engineer to understand better where the noise come from, and eliminate the noise source at the place it is generated rather than using bulky filter to resist noise flowing to the LISN.

One important concept of the $L C P$ model is that it provides only the Least number of Coupling Paths connected between the terminals of LISN and voltage nodes in the converter. It doesn't show all noise sources and paths in a converter. For example, the input ripple current or mutual inductive coupling effect have not been shown which should be calculated with the original circuit elements and parasitic elements. Nevertheless, it is not difficult to modify the $L C P$ model to incorporate other noise source. 
One obvious noise source which is not shown by the mutual capacitive couple model is mutual inductive coupling. Nevertheless, it is easy to give slight modification on the LCP model to consider both the mutual capacitive and mutual inductive coupling effect. The following figure shows also the mutual inductive coupling to the terminals of the LISN through non-contact paths.

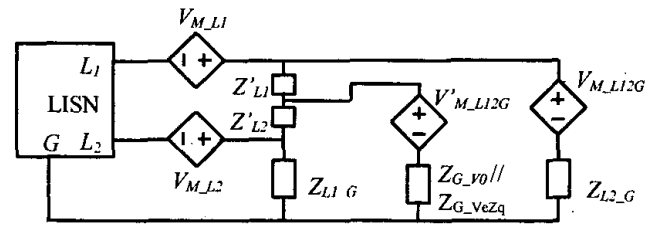

Fig. 2 mutual inductive effect on the terminals of LISN through $L C P$ model.

Similar to mutual capacitive coupling, the equivalent voltages $V_{M L 1}, V_{M_{L 22}}, V_{M_{L 12 G}}^{\prime}$ and $V_{M_{L L 12 G}}$ induced in the four loops of the AC equivalent of the $L C P$ model. Mutual inductive coupling can be related as an equivalent current source $I_{e q}$ and equivalent mutual impedance $Z_{M_{-} L_{-}{ }_{-} \text {eq }}$, $Z_{M_{-} L 2 \text { Ieq }}, Z_{\text {M_L12G }}^{\prime}$, and $Z_{M_{L} \text { L12G_Ieq }}$ such that

$$
\begin{aligned}
& V_{M_{-} L 1}=I_{e q} Z_{M_{-} L 1_{-} I e q} \\
& V_{M_{-} L 2}=I_{e q} Z_{M_{-} L 2_{-} I e q} \\
& V_{M_{-} L 12 G}=I_{e q} Z_{M_{-} L 12 G_{-} I e q} \\
& V_{M_{-} L 12 G}=I_{e q} Z_{M_{-} L 12 G_{-} \mathrm{leq}}
\end{aligned}
$$

Again, $I_{\text {eq, }} Z_{M_{-} L I_{-} G_{-} I e q}, Z_{M_{-} L I l_{-} \text {leq }}, Z_{M_{-} L 12 G_{-} I e q}^{\prime}$ and $Z_{M_{-} L I 2 G_{-} \text {leq }}$ are parameters where one arbitrary defines while others can be solved from Eqn. (7) ,(8), (9) and (10). Impedance $Z_{L I}^{\prime}$ and $Z_{L 2}^{\prime}$ are the equivalent parallel impedance of $Z_{L 1}, Z_{L L} V e q$, $Z_{L I_{-} V 0}$ and $Z_{L 2}, Z_{L Z_{-} V e q}, Z_{L 2_{-} V 0}$ respectively.

The $L C P$ model is now completed to explain all kinds of non-contact noise generated between the converter and the LISN. The noise or input ripple current generated from the converter itself can be modelled as another noise current source connected between input line $L_{1}$ and $L_{2}$. Although the detail formation of the current source is not discussed, it is widely discussed in a lot of other articles[1], [3].

\section{II.II Physical View}

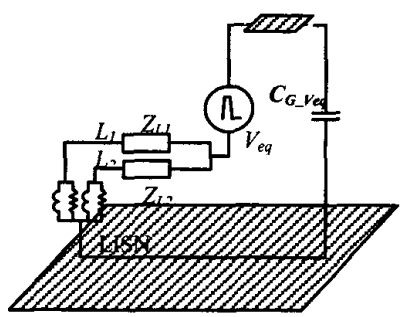

(a)

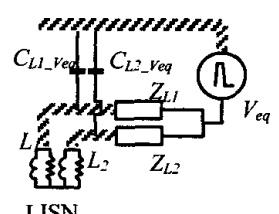

(b)

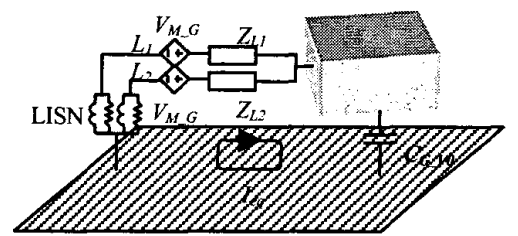

(c)

Fig. 3 Physical meaning of each paths a)path containing $C_{G_{-} V e q}$ b)path containing $\left.C_{L I_{-} V e q}, C_{L Z_{-} V e q} \mathrm{c}\right)$ path containing $C_{G_{-} \text {Vo }}$.

Imagine that there is a boost converter having a dominant voltage source $V_{e q}$ across the drain source of the main switching MOSFET. The $L C P$ paths can be physically explained.

Considering capacitance $C_{G_{-} V e q}$ by approximating $V_{e q}$ to be a dominated noise source having potential $V_{k}$ with relation $V_{e q}=V_{e q}^{\prime}-V_{0}$, where $V_{0}$ is the potential of the $0 V$ reference of the circuit

$$
V_{e q}^{\prime}=V_{k} \text {, for } 0<k<n,
$$

as $V_{k}$ is dominant

$$
V_{e q}^{\prime} \gg V_{i} \text { and } V_{i}=0, \quad \text { for } i \neq k
$$

according to (3),(6),(12), $C_{G_{Y} \text { Veq }}$ is reasonably chosen as the capacitance between the dominant node and earth. Or

$$
C_{G_{-} v_{e q}}=C_{G_{-} k} \text {. }
$$

Capacitor $C_{G_{-} V e q}$ can be understood as the total equivalent capacitance between the dominant switching voltage nodes to earth. It can be approximated as the "hot node" as shown in Fig. 3a, which is capacitance to earth. This has been widely used in analysis of EMI behavior in a switching converter.

Similar to the analysis of $C_{G_{-} V e q}$, capacitors $C_{L_{-} V_{e q}}$ and $C_{L 2_{-} V_{e q}}$ can be understood as the total equivalent capacitance between the dominant switching voltage nodes to the two input lines $L_{1}$ and $L_{2}$, it can be approximated as the "hot node" capacitance to two input lines as shown in Fig. 3b, but this is not commonly discussed[8].

Capacitance $C_{G_{-} v 0}$ can be found by keeping the above assumption of dominant noise source. From (3),(6),(12), and, all other nodes are virtually $\mathrm{AC}$ connected to $V_{\theta}$, or

$$
C_{G_{-} V 0}=\sum_{i=0}^{i \neq k=n} C_{G_{-} i},
$$


Capacitor $C_{G_{-} v \theta}$ now can be understood as the total equivalent capacitance between the "zero" voltage nodes to earth. It can be approximated as the total capacitance of whole converter to earth as shown in Fig. 3c. It has a much higher value or much lower impedance to produce significant effect by the mutual inductive voltage $V_{M_{-} L 1}$, and $V_{M_{-} L 2}$ on input wires, but only a few engineer consider this path.

Capacitor $C_{L I V 0}$ and $C_{L 2} v 0$ are shown as it is obviously parallel and integrated with input equivalent impedance $Z_{L I}$ and $Z_{L 2}$.

\section{ILLUSTRATION AND EXPERIMENT}

\section{III.I Illustration}

In order to make the idea presented so far more comprehensible, the illustration below gives an idea of noise caused by each paths in the minimum coupling paths model. Simulation will be shown and a proper example with reasonable parameter in a real switching power supply will be presented.

It is reasonable to define $V_{e q}$ as a voltage pulse with $400 \mathrm{~V}$ peak, $50 \mathrm{~ns}$ rise and fall time, switching frequency of $150 \mathrm{kHz}$, and duty cycle is 0.2 . This definition can give a good approximation when the defined voltage source is the dominant switching voltage node of a converter.

The arbitrary current source $I_{e q}$ is reasonable to be defined as series $L, R, C$ impedance connected parallel with the voltage source $V_{e q}$. Choose $R, L, C$ as the parasitic elements with values $R=0.05 \Omega, L=500 \mathrm{nH}, C=100 \mathrm{pf}$. These values give a good illustration of current loop in a switching converter, e.g. loop form by two winding in the main power transformer where $C$ is capacitance between two winding, $R$ and $L$ is the equivalent series resistance and inductance of loop containing the two winding.

Once $V_{e q}$ and $I_{e q}$ are defined, the corresponding coupling capacitance can be deduced.

Assuming the area of the dominant node is a thin copper trace with width of $W=0.2 \mathrm{~cm}$, length $l=2 \mathrm{~cm}$, thickness $t=$ $0.01 \mathrm{~cm}$ and separate to earth with distance $R=40 \mathrm{~cm}$. The capacitance [1] can be approximated by

$$
C_{\text {plate_plate }_{\text {pla }}}=0.085 \mathrm{Wl} \mathrm{pF},
$$

hence

$$
C_{G_{-} \text {Veq }}=0.035 \mathrm{pF}
$$

Let the dominant trace are parallel with the PCB trace of Line 1 and Line 2 with $5 \mathrm{~cm}$ and $5.4 \mathrm{~cm}$ by distance. Both PCB traces of Line 1 and Line 2 have the same width and length of the dominated trace. According to [3]

$$
C_{\text {trace_trace }}=\frac{14\left(\varepsilon_{r}+1\right)}{\ln \left(\frac{\pi s}{W+t}\right)},
$$

Similarly, capacitance $C_{L L_{-} V e q}$ and $C_{L_{2} V_{e q}}$ can be found out as,

$$
C_{L I_{-} \text {Veq }}=0.322 p f, C_{L 2_{-} V e q}=0.316 p f \text {. }
$$

Assuming the whole converter is equivalent to a sphere with $r=5 \mathrm{~cm}$ radius, as the self capacitance of a sphere is

$$
C_{\text {sphere }}=\frac{4 \pi 0.085}{\frac{1}{r}} p f,
$$

or

$$
C_{G_{-} v 0}=5.5 p f
$$

The last two unknowns are capacitors $C_{L I_{-} V 0}$ and $C_{L Z_{-} V 0}$. Although it can be found by using the same approximation as used in finding $C_{G_{-} v 0}$, which mean the capacitance is the input traces to the whole converter capacitance. However, no suitable close form expression the author can be found to give a result. Nevertheless, the capacitance is absorbed in the input impedance's parallel stray capacitance.

Input impedance of the converter can be assumed as inductive and unbalance for more generalized conditions, e.g.

$$
Z_{L 1}=100 u H+0.5 \Omega / / 25 p f, Z_{L 2}=50 u H+0.3 \Omega / / 17 p F
$$

Assuming the loop formed by $I_{e q}$ is an equivalent circle with $r=0.8 \mathrm{~cm}$, line 1 and line 2 is a 1 meter long cable. The mutual inductance between a circle loop with radius $r \mathrm{~cm}$ separated by a distance $R \mathrm{~cm}$ and a long straight wire is

$$
M_{\text {line_loop }}=u_{0}\left[\frac{R}{100}-\sqrt{\left(\frac{R}{100}\right)^{2}-\left(\frac{r}{100}\right)^{2}}\right],
$$

As the length of input cable is much greater than the radius of the loop formed by $I_{e q}$, the mutual inductance between the equivalent noise loop and line 1 or line 2 when $r$ $=0.8 \mathrm{~cm}, R=2 \mathrm{~cm}$ can be approximated as

$$
M_{L L_{-} G_{-} \text {leq }}=M_{L 2_{-} G_{\text {_leq }}}=2 \mathrm{nH}
$$

The mutual inductance between two concentric loops is given below. $N_{1}$ is the number of turns of the first loop, $r_{l}$ the radius of first loop, $N_{2}$ the number of turn of second loop, $r_{2}$ the radius of second loop, and $R_{s}$ is the separation of two loop measured at center. The mutual inductance is

$$
M_{\text {loop_loop }}=\frac{u_{o} N_{1} N_{2} r_{1}{ }^{2} r_{2}{ }^{2}}{200 R_{s}{ }^{3}},
$$

Estimate that the loop with $r_{2}=0.8 \mathrm{~cm}$ formed by the PCB trace of line 1 and line 2 form a co-planar and concentric loop with loop of $I_{e q}$ with separation $R=5 \mathrm{~cm}$. The mutual inductance between them can be approximated as,

$$
M_{L 1 \_L 2 \_l e q}=0.065 n H
$$

After all, an approximated $L C P$ model can be formed to estimate the noise generated from each path. 


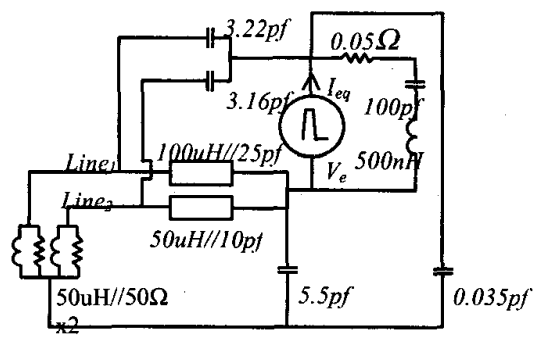

(a)

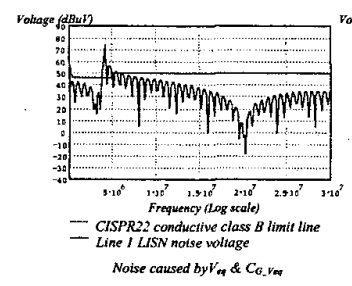

(b)

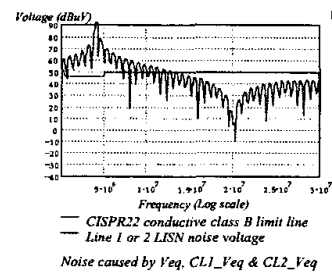

(d)

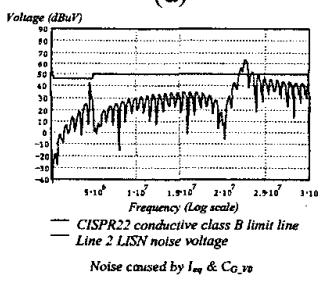

(f)

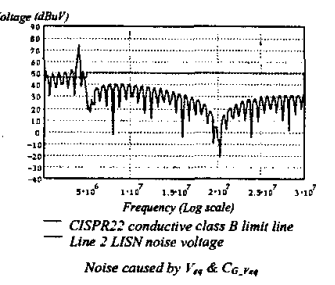

(c)

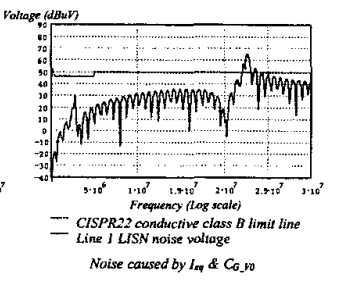

(e)

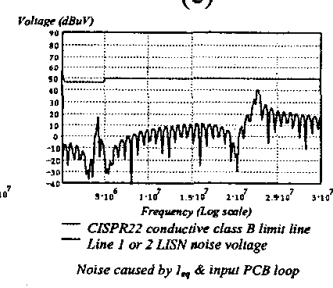

(g)
Fig. 4 Illustration of noise caused by each path a) $L C P$ model of an illustrated example b) line 1 LISN noise when $C_{G_{-} V e q}$ is considered only c) line 2 LISN noise when $C_{G_{-} V e q}$ is considered only d) line 1 or 2 LISN noise voltage when $C_{L I_{-} \text {Veq }}$ and $C_{L 2 \_ \text {req }}$ is considered only e) line 1 LISN noise voltage when loop form by $C_{G_{-} V O}$ is considered only f) line 2 LISN noise voltage when loop form by $C_{G_{-}}$o is considered only g) line 1 or 2 LISN voltage when loop form by PCB trace of line 1 and line 2 only.

Simulations show that each coupling path can produce substantial noise at the LISN, especially at the resonate points. Inherently that there exist a lot of inductors and capacitors inside and around a switching converter, it has good reason to believe the $L C P$ model is a necessary model and requires minimum work.

\section{III.II Lump elements verification}

$L C P$ model gives an indication of minimum scale of circuit which should be considered but it is still complicated and doesn't give direct EMI result. Parameters like mutual capacitance between nodes, mutual inductance between loops and the impedance inside a converter have to make known before finding out the equivalent $L C P$ model. A lot of parameters, e.g. inductance, resistance or capacitance are frequency dependent and involve 3D issues. After all, it is never a simple task to predict EMI result of a switching converter. Again the LCP model shows the simplest form but things are still not easy. Nevertheless, the $L C P$ model is very good for diagnosis and understanding purpose.

The work does not aim at simulation of a particular converter EMI emission but aim at proving the more basic assumption of using lump element theory to model EMI. Predicting the EMI of a particle converter is a second level job where accuracy depends on how accurate the corresponding parameters can be found. Two fundamental experiments about the LCP model, mutual capacitive coupling and mutual inductive coupling, were done to show the applicability of lump elements approach.

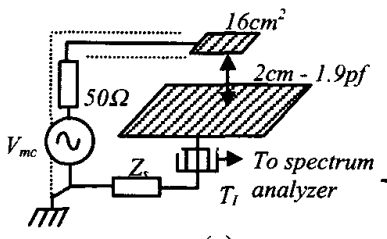

(a)

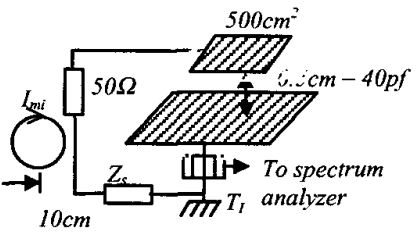

(b)

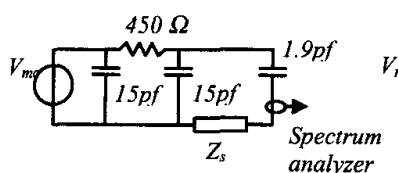

(c)

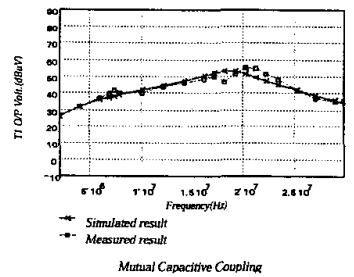

(e)

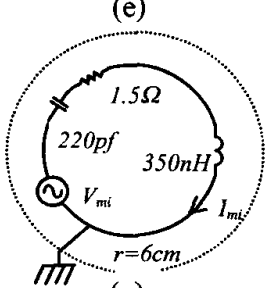

(g)

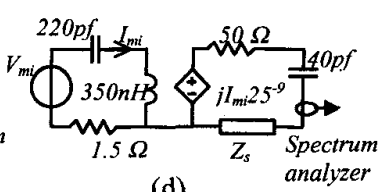

(d)

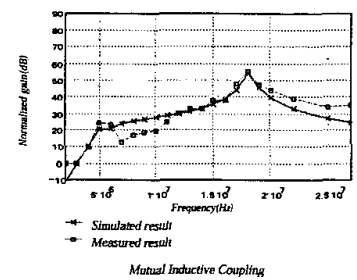

(f)

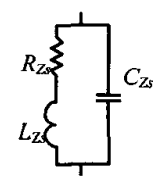

(h)
Fig. 5 Lump elements model verification a) mutual capacitive coupling experimental setup b) mutual inductive 
coupling experimental setup c) equivalent circuit of Fig. 5a, d) equivalent circuit of Fig. $5 \mathrm{~b}$ e) equivalent circuit of current source $I_{m i}$ f)simulated and measured result of Fig. 5a, g)simulated and measured result of Fig. $5 \mathrm{~b}, \mathrm{~h}$ ) equivalent model of impedance $Z_{s}$

Fig. 5a shows the experimental setup which shows the basic physical mutual capacitive coupling model as in Fig. 3a and Fig. 3b. Fig. 5c shows the equivalent circuit. Associated parameters are $V_{m c}=1 \mathrm{Vpeak}$, the output shielded cable has a $30 \mathrm{pf}$ capacitance and series resistance of $400 \Omega$, capacitance between plates is calculated by capacitor modeling software as $1.9 \mathrm{pf}$.

Fig. $5 b$ shows the experimental setup which shows the basic physical mutual inductive coupling model as in Fig. 3c with capacitance between two plates measured by impedance analyzer HP4194 as 40pf. Fig. 5g shows the equivalent of the exciting loop. The mutual inductance is calculated by the relation between a line and a loop and is equal to $25 \mathrm{nH} . V_{m i}$ is approximately $0.5 \mathrm{~V}$, and vary with frequency, nevertheless normalized gain against the $T_{I} \mathrm{O} / \mathrm{P}$ voltage and $V_{m i}$ is used to give sensible result. Fig. $5 \mathrm{~d}$ shows the equivalent circuit.

The current transformer $T_{l}$ has a conversion ratio of $1 u \mathrm{~A}$ to $0.5 \mathrm{uV}$ into a $50 \Omega \mathrm{load}$, the coupling current are read out by the spectrum analyzer at the position as shown in Fig. 5a and Fig. 5b. The voltage source $V_{m i}$ changes with frequency, especially when the current loop resonates and becomes virtually short circuit.

The series impedance $Z_{s}$ is a wire wound inductor with equivalent circuit as shown in Fig. $5 \mathrm{~h}$, nevertheless, the parameters are frequency dependent. Values below $5 \mathrm{MHz}$ are $L_{Z s}=13 u H, R_{Z s}=7 \Omega$ and $C_{Z s}=5 p f$, for frequency above $5 \mathrm{MHz}$ and below $30 \mathrm{MHz}$, the values are $L_{\mathrm{Zs}}=11.4 u H, R_{\mathrm{Zs}}=$ $333 \Omega$ and $C_{Z s}=1.5 p f$

Fig. 5e and Fig. 5f show comparison of measured results and simulated results using lump elements for frequencies below $30 \mathrm{MHz}$, which is the frequency range for conducted EMI. Results show that lump element model give reasonable accuracy for explaining coupling effect, simulated result compares very well with the measured data. At such low level of voltage measurement, it is well understood that the whole system error [1] can be as large as $10 \mathrm{~dB}$. It shows it is useful to use lump element to analysis non-contact EMI. The rest of job is to find out the value of equivalent coupling capacitance and impedance in order to demonstrate the effect of each coupling path. Eventually it turns out to be a combination of work between physics and electronics.

\section{CONCLUSIONS}

A simplified equivalent model gives a set of Least Coupling Paths which consists of equivalent capacitors between terminals of LISN and the equivalent voltage source of the converter. Each path is important and should not be ignored without preliminary consideration. Consistent analysis of EMI is presented. Lump element model is proven to have enough accuracy for frequencies below $30 \mathrm{MHz}$. Both capacitive and inductive coupling may produce significant noise at the LISN or noise current flow through the input cable. The $L C P$ model is good for EMI explanation and diagnosis, and hence pave ways to eliminate effect of the noise source.

\section{REFERENCE}

[1] Tim William, EMC for Product Designers, ButterworthHeinemann Ltd. 1994

[2] Jeffrrey P.Mill, Electro-magnetic Interference Reduction in Electronic Systems, PTR Prentice Hall, Englewood Cliffs, New Jersey 07632,1993

[3] Mark D. Herema, Designing for Electromagnetic Compatibility, HP11949B Hewlett Packard.

[4] Dongbing Zhang; Dan Chen; Sable, D. Non-intrinsic differential mode noise caused by ground current in an off-line power supply, Power Electronics Specialists Conference, 1998. PESC 98 Record. 29th Annual IEEE Volume: 2,1998 , Page(s): 1131 -1133 vol.2

[5] Wei Zhang; Zhang, M.T.; Lee, F.C.; Roudet, J.; Clavel, E., Conducted EMI analysis of a boost PFC circuit, Applied Power Electronics Conference and Exposition, 1997. APEC '97 Conference Proceedings 1997., Twelfth Annual Volume: 1, 1997 , Page(s): 223 -229 vol.1.

[6] Ferreira, J.A.; Willcock, P.R.; Holm, S.R., Sources, paths and traps of conducted EMI in switch mode circuits, Industry Applications Conference, 1997., Thirty-Second IAS Annual Meeting, IAS '97.,

Conference Record of the 1997 IEEE, Volume: 2, 1997 , Page(s): $1584-1591$ vol.2.

[7] Qing Chen, Electromagnetic interference (EMI) design considerations for a high power AC/DC converter, Power Electronics Specialists Conference, 1998. PESC 98 Record. 29th Annual IEEE Volume: 2, 1998 , Page(s): 1159 -1164 vol.2.

[8] Pong, B.M.H.; Lee, A.C.M., A method to measure EMI due to electric field coupling on PCB, Power Conversion Conference Nagaoka 1997.,Proceedings of the Volume: 2 , 1997 , Page(s): 1007 -1012 vol.2

[9] Peter Bardos, Predicting the EMC Performance of High Frequency Inverters, APEC2001, proceeding of volume 1, 2001, page(s) $213-219$ Vol. 1 . 